\title{
A multi-particle drift-diffusion model and its application to organic and inorganic electronic device simulation
}

\author{
Daniele Rossi, Francesco Santoni, Matthias Auf der Maur, Member, IEEE and Aldo Di Carlo, Member, \\ IEEE .
}

\begin{abstract}
In this work we present a novel generalized multi-particle drift-diffusion model capable to overcome some limitations imposed by the classic drift-diffusion model by taking into account multiple carrier populations. We demonstrate the model's ability and flexibility in simulating systems from different application contexts. We conclude that the developed multi-particle drift-diffusion model allows to investigate a wide range of complex mechanisms, such as sub-band charge transport calculation or intersystem crossing, that are crucial for the understanding and design of organic and inorganic semiconductor devices largely employed for both photovoltaics and light emitting applications.
\end{abstract}

Index Terms-Band-to-band transition, drift-diffusion, exciton transport, OLED, organic optoelectronic, semiconductor device modeling.

\section{INTRODUCTION}

The traditional approach for semiconductor transport modeling in electronic devices, used extensively both in commercial technology computer aided design (TCAD) software and in academic codes, is based on a set of semi-classical transport equations for an electron and a hole population, assumed each in local thermal equilibrium with the host material. Particle fluxes are then driven solely by gradients of thermodynamic potentials, namely the carriers' electrochemical potentials and the inverse temperature. This model is known as the driftdiffusion model or the van Roosbroeck equations [1], and has been the workhorse of electronic device simulation during the last fifty years. The assumption of local thermal equilibrium implies that the carrier densities can be written as integrals of the product of a Fermi function with some density of states (DOS). Most often, a density of states resulting from a parabolic dispersion, an idealization around the band extrema of a perfectly periodic crystal, is assumed, leading to Fermi integrals, for which analytic approximations exist. As a further

\footnotetext{
Corresponding authors: Matthias Auf der Maur (e-mail: auf.der.maur@ing.uniroma2.it) and Aldo Di Carlo (e-mail: aldo.dicarlo@uniroma2.it)

D. Rossi, M. Auf der Maur, and A. Di Carlo are with the Department of Electronic Engineering, Università di Roma Tor Vergata, Via del Politecnico 1, 00133 Roma, Italy.

F. Santoni was with the Department of Electronic Engineering, University of Roma Tor Vergata, Via del Politecnico 1, 00133 Roma, Italy, now he is with the Department of Engineering, University of Perugia, via G. Duranti, 93, 06125 Perugia, Italy.
}

simplification, usually the Boltzmann approximation is used, so that the densities are given by the familiar exponential expressions.

An alternative approach to describing electronic transport in devices is to directly solve the Boltzmann transport equation (BTE), for example by self-consistent ensemble Monte Carlo technique [2]. While this allows to completely drop any a priori assumption on the occupation probabilities in the phase space, it is a computationally much heavier approach to the problem. Especially in organic electronics, the kinetic Monte Carlo (kMC) technique has found increasing interest during the last years. This approach is particularly suited to describe systems where transport occurs via hopping between localized states. kMC implementations have also been extended to include transport of excitons, which is of particular interest in organic optoelectronics [3], [4].

Multi-particle transport models have been employed since decades in process simulation, like e.g. in Yoshida et al. [5] for modeling of ion implantation. Over the years, several extensions of the van Roosbroeck equations have been developed also for device simulation. These implementations have been designed for specific situations, e.g. as done by Römer et al. [6] for multiple quantum well (MQW) LED simulation, or by Kantner et al. [7] for transport modeling in quantum dot (QD) systems.

The purpose of this work is to formulate and implement an extendible framework for multi-particle drift-diffusion simulations, which is flexible enough to be applied to most devices where such a concept is applicable. For this, we propose a generalization of the semi-classical drift-diffusion based two-carrier transport model, which can overcome some of its limitations and may thus relax the necessity to resort to more expensive approaches like Monte Carlo in a number of relevant device structures. Our modeling approach is based on two ingredients, namely (1) the generalization to more than two carrier populations, each individually assumed in a local thermal equilibrium characterized by a local quasi Fermi level, and (2) a strictly thermodynamically consistent formulation of the transitions between populations, appearing as recombination-generation terms in the coupled system of equations. The model is designed for situations, where the total particle population can be split into sub-populations that are coupled weakly with each other compared to the 


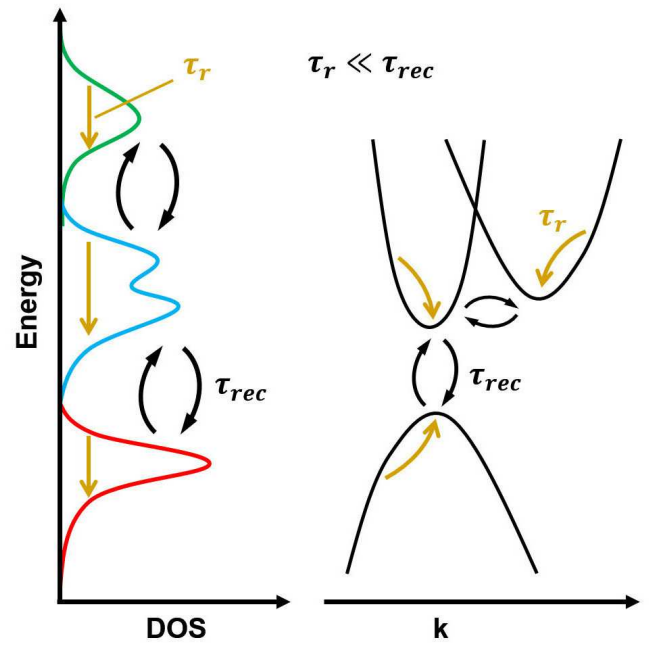

Fig. 1. The basic assumption of the multi-particle drift-diffusion model: several particle populations exist (e.g. in different bands, as on the right), which are in individual local thermal equilibrium and weakly coupled with each other. $\tau_{r}$ and $\tau_{r e c}$ are the characteristic intra-band (relaxation) and inter-band (recombination) scattering times.

relaxation inside the populations, as schematically shown in Fig. 1. The standard drift-diffusion model is a special case of this, with a valence and a conduction band population, each with its own quasi Fermi level, are coupled by interband recombination with a rate that is usually lower than the intra-band scattering rates. This multi-particle drift-diffusion model (mp-DD) improves the flexibility of the drift-diffusion model as such, and it also increases reusability, since the same implementation can be adjusted to different application contexts.

In the following we will describe the salient features of the model and will show a few application examples of our implementation.

\section{THE MODEL}

The multi-particle drift-diffusion model is based on the generalized van Roosbroeck system and allows to define any number of carriers, each one with its own properties as charge, spin and DOS. For simplicity we stick to the stationary case, in which the model reads as [1], [8]

$$
\begin{gathered}
\nabla \cdot\left(\varepsilon_{0} \varepsilon_{r} \nabla \varphi-\mathbf{P}_{\mathbf{0}}\right)=-q \sum_{i} z_{i} n_{i}-q C \\
\nabla \cdot\left(\mu_{i} n_{i} \nabla \phi_{i}\right)=\operatorname{sgn}\left(z_{i}\right) R_{i}, \forall i
\end{gathered}
$$

The Poisson equation (1a) determines the electrostatic potential $\varphi$ from the sum of the charge densities resulting from all charged carrier densities $n_{i}$, and possibly other fixed charge distributions, indicated by $C$, which are not treated as mobile carriers. $q$ and $z_{i}$ in (1a) are the elementary charge and the $i$-th carrier's charge number. We also include explicitly a fixed polarization $\mathbf{P}_{\mathbf{0}}$, which models e.g. spontaneous and piezoelectric polarization in certain materials like III-nitrides [9]. The continuity equations (1b) describe the transport of all carriers included in the system. The carrier fluxes are written in terms of the gradient of the quasi Fermi potentials $\phi_{i}, \mathbf{j}_{i}=$ $-\operatorname{sgn}\left(z_{i}\right) \mu_{i} n_{i} \nabla \phi_{i}$, where $\mu_{i}$ is the carrier mobility, given by a suitable mobility model. The quasi Fermi potentials are related to the electrochemical potentials or quasi Fermi levels via $E_{f, i}=-q \phi_{i}$ [8]. $R_{i}$ is the total net recombination-generation rate of the $i$-th carrier, which in general will depend on different carrier densities and thus couples the different carrier populations. As primary variables in our implementation, we prefer the quasi Fermi potentials over the densities, since this allows to write the equations in a more generic form. The densities $n_{i}$ are then given by the generic expression [10]

$$
n_{i}=\int_{-\infty}^{\infty} D_{i}(E) \frac{1}{\exp \left(\frac{E+\operatorname{sgn}\left(z_{i}\right) q \phi_{i}-z_{i} q \varphi}{k_{B} T}\right) \pm 1} \mathrm{~d} E .
$$

Here, $D_{i}(E)$ is the DOS of the $i$-th carrier, and the \pm in the occupation factor indicates that we allow for BoseEinstein statistics in order to describe also other types of quasiparticles like excitons. Therefore $z_{i}$ can be also 0 . In analogy to chemistry, all recombination rates are written as reactions between different species in the system in the general form [11]:

$$
\begin{aligned}
& \sum_{j} \alpha_{j} n_{j} \rightleftharpoons 0 \rightarrow \sum_{j} \alpha_{j} \phi_{j}=0 \\
& r_{i}=\alpha_{i}\left[1-e^{-\frac{q}{k_{B} T} \sum_{j} \alpha_{j} \phi_{j}}\right] \sum_{\left\{\theta_{j}\right\}} \gamma\left(\left\{\theta_{j}\right\}\right) \\
& \times \prod_{j}\left[\frac{1}{2}\left(1-\operatorname{sgn}\left(\alpha_{j}\right)\right) \pm f_{j}\left(\theta_{j}\right)\right]^{\left|\alpha_{j}\right|} .
\end{aligned}
$$

Equation (3a) shows the reaction and corresponding thermodynamic equilibrium condition in the isothermal case. $\alpha_{j}$ is an integer giving the stoichiometric weight of the $j$-th particle in the reaction, i.e. how many particles of population $i$ are involved. Its sign distinguishes whether the particle is generated or destructed in the reaction. Equation (3b) is based on the formulation of the interband scattering terms appearing in the BTE [12], using however for the distribution functions the equilibrium expressions, and generalizing to any number of particles involved in the reaction. It gives the general expression for the net recombination rate of the $i$-th carrier for a specific recombination process, which contributes to the total net recombination rate $R_{i}=\sum r_{i}$ entering (1b). The $\left\{\theta_{j}\right\}$ indicates the set of degree of freedoms of the different particles, like e.g. crystal momentum or spin. Note that the occupation factors $f$ in general depend on their respective degree of freedoms via the energy levels. The sign in front of $f$ depends on the sign of $\alpha$ and the type of statistics. A distinctive and important feature of the model of (1)-(3) is its inherently thermodynamically consistency. In fact, we can associate thermodynamic equilibrium with $\phi_{j}=0$, which leads to both zero carrier flux and vanishing net recombination. Therefore, the solution of the non-linear Poisson equation with $\phi_{j}=0$ provides the unique equilibrium solution of the system (1). From (3b) we can derive the necessary recombination 


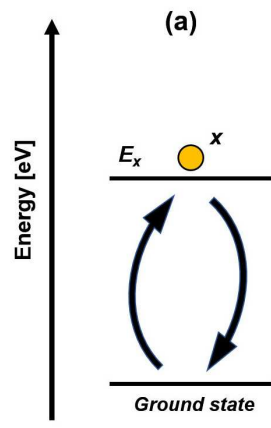

(b)

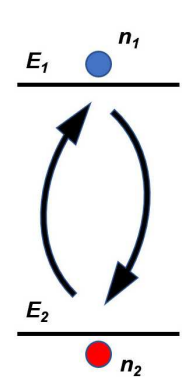

(c)

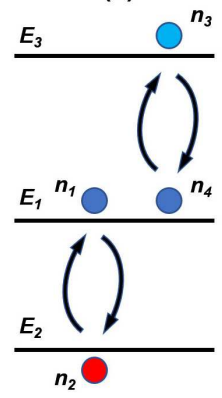

Fig. 2. Some common recombination mechanisms. (a) One-carrier relaxation process, like exciton decay; (b) two-carrier process like radiative recombination or band-to-band transfer; (c) three-carrier process like Auger recombination.

models, involving any number of carriers. Some of the most common are schematically shown in Fig. 2. In the following we will give a few examples and show that the generic formulation indeed reduces to the well-known formulas used with the Van Roosbroeck system.

\section{A. Single carrier mechanisms}

Single carrier mechanisms (Fig. 2a) such as non-radiative and radiative decay of excitons are relevant e.g. in organic light emitting diodes (OLEDs) or organic photovoltaics (OPV). The reaction for exciton density $(x)$ becomes as follows:

$$
\begin{aligned}
& x \rightleftharpoons 0 \rightarrow \phi_{x}=0 \\
& r(\theta)=\gamma(\theta) f_{\theta}\left(\phi_{x}\right)-\gamma^{\prime}(\theta)\left[1+f_{\theta}\left(\phi_{x}\right)\right] \\
& \gamma^{\prime}(\theta)=\gamma(\theta) \exp \left(-\frac{E_{x}(\theta)}{k_{B} T}\right)
\end{aligned}
$$

As before, $\theta$ parametrizes the internal degrees of freedom, and the last equation results from the detailed balance [10], requiring that at equilibrium forward and backward reaction must be equal. $E_{x}(\theta)$ is the energy level of the exciton in state $\theta$. Note that we treat excitons as quasi particles that obey BoseEinstein statistics, therefore a term $1+f_{\theta}$ appears in (4b). It can be expected that this only holds for not too high exciton densities [13]. Substituting (4c) in (4b) and summing over the internal degrees of freedom, we obtain the net recombination rate as

$$
R=\left[1-\exp \left(\frac{q \phi_{x}}{k_{B} T}\right)\right] \sum_{\theta} \gamma(\theta) f_{\theta}\left(\phi_{x}\right)
$$

Equation (5) can be simplified, when we can assume that $\gamma$ is independent on $\theta$. In that case, the sum of $f_{\theta}$ over all $\theta$ evaluates to the density $x$, so that

$$
R_{\text {decay }}=\frac{x}{\tau}\left[1-\exp \left(\frac{q \phi_{x}}{k_{B} T}\right)\right]
$$

where we have defined the decay time as $\tau=1 / \gamma$. Note that whenever the Boltzmann approximation can be used for the density $x$ the last formula can be rewritten as $R_{\text {decay }}=(x-$ $\left.x_{0}\right) / \tau$, with $x_{0}$ the equilibrium density. This is the familiar formula for a decay process in relaxation time approximation [14].

\section{B. Two-carrier mechanisms}

Two-carrier mechanisms which involve charged carriers (same or different species) such as direct (DIR) or bandto-band (BB) recombinations [15], or neutral carriers such as triplet-to-triplet annihilation (TTA) [16], up to combined species such as triplet-to-polaron quenching (TPQ) [17] are relevant in both organic and inorganic optoelectronic devices. Considering two carriers $\left(n_{1}\right.$ and $\left.n_{2}\right)$ which belong to different energy levels $\left(E_{1}\right.$ and $\left.E_{2}\right)$ as shown in Fig. 2(b), the corresponding reaction can be written as follows:

$$
\begin{aligned}
& n_{1}+n_{2} \rightleftharpoons 0 \rightarrow \phi_{n_{1}}=\phi_{n_{2}} \\
& r\left(\theta_{1}, \theta_{2}\right)=\gamma_{1,2} f_{\theta_{1}}\left(1-f_{\theta_{2}}\right)-\gamma_{2,1} f_{\theta_{2}}\left(1-f_{\theta_{1}}\right) \\
& \gamma\left(\theta_{1}, \theta_{2}\right)=\gamma_{1,2}\left(\theta_{1}, \theta_{2}\right)=\gamma_{2,1}\left(\theta_{1}, \theta_{2}\right) \exp \left(-\frac{E_{2}-E_{1}}{k_{B} T}\right) .
\end{aligned}
$$

Equation (7b) describes forward and backward reaction between states $\theta_{1}$ and $\theta_{2}$, where $f_{\theta_{1}}$ and $f_{\theta_{2}}$ depend respectively on $\phi_{n_{1}}$ and $\phi_{n_{2}}$. In case $E_{1}>E_{2}, \gamma_{1,2}$ is the emission coefficient, while $\gamma_{2,1}$ is the capture coefficient, and they are related to each other by (7c). According to both Fermi-Dirac (FD) or Bose-Einstein (BE) and substituting (7c) in (7b) we can obtain a generic valid formulation for two carriers, whether they are either different species or the same.

$$
\begin{array}{r}
r=\sum_{\theta_{1}, \theta_{2}} \gamma\left(\theta_{1}, \theta_{2}\right) \exp \left(-\frac{E_{1}-E_{2}}{k_{B} T}\right) f_{\theta_{1}}\left(\phi_{n_{1}}\right)\left[1-f_{\theta_{2}}\left(\phi_{n_{2}}\right)\right] \\
\times\left[1-\exp \left(\frac{q\left(\phi_{n_{2}}-\phi_{n_{1}}\right)}{k_{B} T}\right)\right]
\end{array}
$$

As an example, considering electrons $\left(n_{1}\right)$ and holes $\left(n_{2}\right)$ in a semiconductor crystal with parabolic bands, the sum in (8) becomes an integral over the crystal momenta $k_{1}$ and $k_{2}$.

$$
\begin{aligned}
r=\left[1-\exp \left(\frac{q\left(\phi_{p}-\phi_{n}\right)}{k_{B} T}\right)\right] \frac{1}{(2 \pi)^{6}} \\
\quad \times \int \tilde{\gamma}\left(k_{1}, k_{2}\right) f_{1}\left(1-f_{2}\right) d k_{1} d k_{2}
\end{aligned}
$$

Here, the transitions are explicitly dependent on the crystal momenta as $\tilde{\gamma}\left(k_{1}, k_{2}\right)$. Then, using Boltzmann approximation we obtain the well-known formula for direct recombination as

$$
R_{D I R}=C_{D I R} n p\left[1-\exp \left(\frac{q\left(\phi_{p}-\phi_{n}\right)}{k_{B} T}\right)\right]
$$

where $C_{D I R}$ is the radiative rate parameter [15]. 


\section{Three-carriers mechanisms}

Although mechanisms such as eeh and hhe Auger recombinations involve three carriers of different species, the reaction in (11b) includes the initial states $\theta_{1}, \theta_{2}$ and $\theta_{4}$ and the final state $\theta_{3}$ of the excited carrier $n_{3}$ as shown in Fig. 2(c).

$$
\begin{aligned}
& 2 n_{1}+n_{2} \rightleftharpoons n_{3} \rightarrow 2 \phi_{n_{1}}=\phi_{n_{2}}+\phi_{n_{3}} \\
& r\left(\theta_{1}, \theta_{2}, \theta_{3}, \theta_{4}\right)=\gamma f_{\theta_{1}}\left(1-f_{\theta_{2}}\right) f_{\theta_{4}}\left(1-f_{\theta_{3}}\right) \\
& -\gamma^{\prime} f_{\theta_{2}}\left(1-f_{\theta_{1}}\right) f_{\theta_{3}}\left(1-f_{\theta_{4}}\right)
\end{aligned}
$$

We obtain the following formula for the Auger recombination rate of carrier $i$

$$
\begin{aligned}
r_{i}=\alpha_{i} \sum_{\theta_{1}, \theta_{2}, \theta_{3}, \theta_{4}} & \gamma\left(\theta_{1}, \theta_{2}, \theta_{3}, \theta_{4}\right) f_{\theta_{1}}\left(1-f_{\theta_{2}}\right) f_{\theta_{4}}\left(1-f_{\theta_{3}}\right) \\
\times & {\left[1-\exp \left(-\frac{q\left(\phi_{n_{3}}-2 \phi_{n_{1}}+\phi_{n_{2}}\right)}{k_{B} T}\right)\right] . }
\end{aligned}
$$

Here, the factor $\alpha_{i}$ accounts for the fact, that two carriers of population 1 disappear in the reaction $\left(\alpha_{1}=2\right)$, while only one of population 2 disappears $\left(\alpha_{2}=1\right)$ and one of population 3 is created $\left(\alpha_{3}=-1\right)$.

As in the example before, in the case we can use the Boltzmann approximation, (12) simplifies to

$$
\begin{aligned}
R_{\text {Auger }}=C_{\text {Auger }} n^{2} p\left(1-\frac{n^{*}}{N^{*}}\right) \\
\times\left[1-\exp \left(-\frac{q\left(\phi_{n^{*}}-2 \phi_{n}+\phi_{p}\right)}{k_{B} T}\right)\right] .
\end{aligned}
$$

Here, the star indicates the quantities of the carriers in the higher band, and the second carrier is described as a hole. The factor $\left(1-n^{*} / N^{*}\right)$ models empirically the dependency on the occupation of the final electronic states. It is obtained from the $\left(1-f_{\theta_{3}}\right)$ term in (12), using the Boltzmann approximation, so that $N^{*}$ here is the effective density of states for population 3 . This approximated model describes Auger recombination and reduces to the standard model when $\phi_{n^{*}}=\phi_{n}$ and $n^{*} \ll N^{*}$ is assumed [18].

\section{Results And discussion}

The model described in section II has been implemented using the Galerkin finite element method (FEM) in the simulation software TiberCAD [19]. A Newton method with line search is used for the solution of the non-linear equations.

In the following we present a few simulation examples from contexts, where a multi-particle drift-diffusion model can be useful.

\section{A. Thermionic emission}

As a first example, we show that our mp-DD reproduces the current across a heterointerface or at a Schottky contact, including thermionic emission [20]. We approximate a Schottky contact by substituting the metal with a highly doped semiconductor and a band offset with respect to the semiconductor. This allows comparing the analytical formula for a Schottky contact with thermionic emission only to the numerical results. The current density across a Schottky contact for a semiconductor with parabolic bands is given by [20]

$$
J_{M S}=\lambda_{R} A^{*} T^{2} \exp \left(-\frac{\phi_{B}}{k_{B} T}\right)\left[\exp \left(\frac{q V_{a}}{k_{B} T}\right)-1\right],
$$

where, $T$ is the temperature, $\lambda_{R}$ is a material-specific correction factor and $A^{*}$ is the Richardson constant that depends on the electron effective mass $\left(m^{*}\right)$ and it is given by

$$
A^{*}=\frac{4 \pi q m^{*} k_{B}^{2}}{h^{3}} .
$$

For the simulation we assume a silicon Schottky contact with barrier of $\phi_{B}=0.8 \mathrm{eV}$. We consider $1 \mu \mathrm{m}$ thick lightly n-doped Si bulk with $N_{d}^{-}=10^{17} \mathrm{~cm}^{-3}$. The second contact is modeled as an ideal ohmic contact. First we simulate the device using the standard DD approach, where the Schottky contact is modeled by appropriate boundary conditions.

With the mp-DD it is possible to describe the reaction at the metal-semiconductor interface considering different population for metal and semiconductor. To do this we slightly modify the structure including at the anode side an additional $3 \mathrm{~nm}$ thick layer $\left(\Delta x_{m}\right)$, highly doped with $N_{d}^{-}=10^{20}$ $\mathrm{cm}^{-3}$. In the first layer we define the semiconductor carriers $(n)$, while in the additional layer only the contact material's electrons $\left(n^{\prime}\right)$ are present. The Schottky barrier is implemented by setting an energy gap between the band edges of the two electron populations, with a value of $\Delta E_{M S}=0.86 \mathrm{eV}$. This value is chosen such that the effective barrier between the semiconductor conduction band edge and the quasi Fermi level in the contact material results to be $0.8 \mathrm{eV}$ as required. The corresponding energy band profile near the anode is shown in Fig. 3(a). At semiconductor-metal interface (MS), the electron populations are coupled to each other through an interfacial two carrier band-to-band process with rate constant $C_{n n^{\prime}}$.

$$
R_{n n^{\prime}}=C_{n n^{\prime}} n\left(1-\frac{n^{\prime}}{N^{\prime}}\right)\left[\exp \left(\frac{E_{f, n^{\prime}}-E_{f, n}}{k_{B} T}\right)-1\right] \text {. }
$$

By appropriately choosing $C_{n n^{\prime}}$, in this case $C_{n n^{\prime}}=5.1 \times$ $10^{6} \mathrm{~cm} \cdot \mathrm{s}^{-1}$, we can match exactly the result of the standard DD model, as shown in Fig. 3(b). This parameter controls the effective charge transfer between the contact and the semiconductor, and can therefore be related to the parameters in (14). The current calculated with the mp-DD model is obtained as

$$
\begin{aligned}
J_{M S}=q R_{n n^{\prime}} \simeq q C_{n n^{\prime}} N_{C} & \left(1-\frac{n^{\prime}}{N^{\prime}}\right) \times \\
& {\left[\exp \left(\frac{E_{f, n^{\prime}}-E_{f, n}}{k_{B} T}\right)-1\right] . }
\end{aligned}
$$

Comparing this expression for reverse bias with (14) and considering that $V_{a}=E_{f, n}-E_{f, n^{\prime}}$, we obtain 


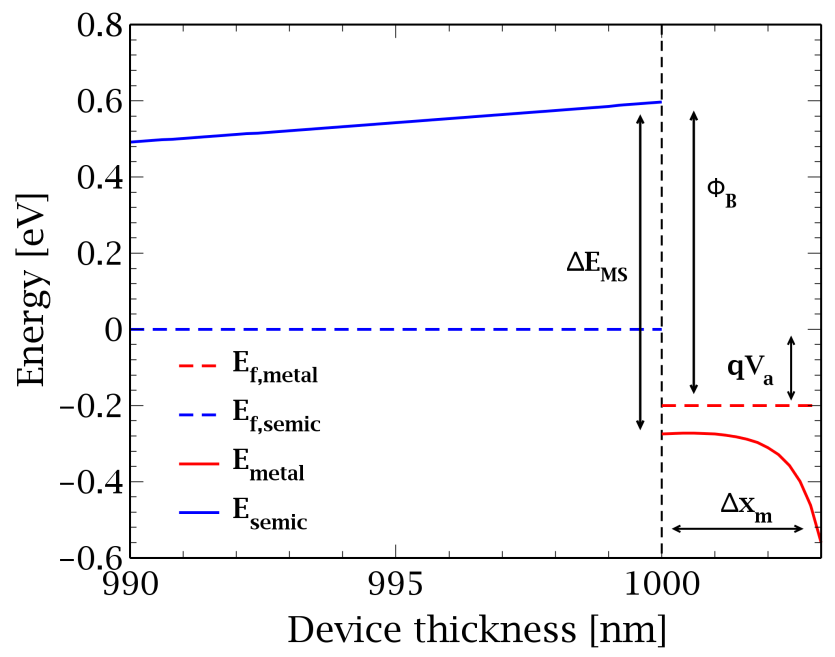

(a)

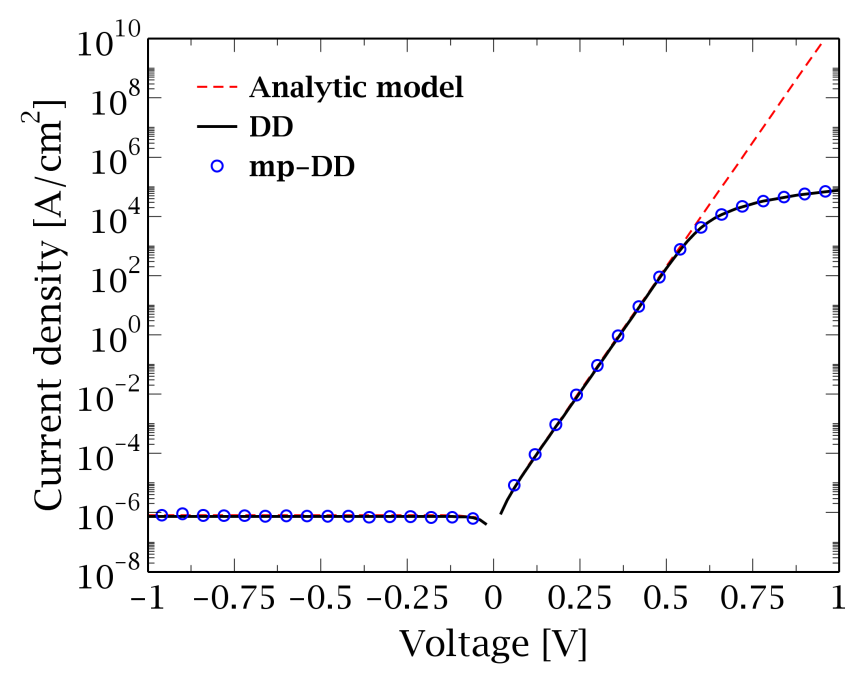

(b)

Fig. 3. (a) Energy band diagram of a silicon Schottky barrier with $\phi_{B}=$ $0.8 \mathrm{eV}$ and $N_{d}^{-}=10^{17} \mathrm{~cm}^{-3}$ at $V_{a}=0.2 \mathrm{~V}$ operation; (b) JV curves comparison of the analytic model (dashed red), classical DD (blue dots) and $\mathrm{mp}$-DD (black).

$$
C_{n n^{\prime}} \simeq \frac{\lambda_{R} A^{*} T^{2}}{q N_{C}},
$$

from which we obtain $C_{n n^{\prime}}=5.0541 \times 10^{6} \mathrm{~cm} \cdot \mathrm{s}^{-1}$, thus in agreement with the value reported above used for the fitting. Therefore, the two models are equivalent in terms of the description of the thermionic emission current at reverse bias. Note that we could formulate an alternative model for the MS interface by introducing an overlapping thin layer $\left(\Delta x_{i n t}\right)$, within which semiconductor and metal electrons coexist and are coupled by a rate constant $C_{n n^{\prime}}$. In this case, the current in (17) would be obtained by integrating the rate $R_{n n^{\prime}}$ over the overlapping region $\Delta x_{i n t}$.

\section{B. Intermediate Band Solar Cells}

The intermediate band solar cell (IBSC) is one of the third generation photovoltaic concepts that potentially allows to increase the ideal maximum power conversion efficiency of conventional single bandgap solar cells, from 40.7\% [21] up to $63.2 \%$ [22]. This is possible by adding an intermediate band (IB) within the forbidden bandgap of the absorbing material of a solar cell, providing an additional photogeneration of carriers by two-step absorption of sub-bandgap photons promoting electrons from the valence band (VB) to the intermediate band and from there to the conduction band (CB). Ideally this process should allow increasing the short-circuit current $\left(J_{S C}\right)$ maintaining at the same time a high open-circuit voltage $\left(V_{O C}\right)$. The IB is typically obtained by growing quantum dot (QD) arrays in a semiconductor or by simply adding metal atoms as dopant in the active material [23].

With the aim to show the model's ability to calculate the charge transport within an intermediate band, we perform simulations on a GaAs-based p-i-n IBSC [24]. The doping densities set in the p-emitter and n-base are, respectively, $N_{a}^{+}=7 \times 10^{16} \mathrm{~cm}^{-3}$ and $N_{d}^{-}=5 \times 10^{16} \mathrm{~cm}^{-3}$, while all material parameters used are referred to $\mathrm{GaAs}$ at 300 K [25]. Simulations account for defect-mediated (ShockleyRead-Hall) recombination, with $\tau_{n}=5 \times 10^{-7} \mathrm{~s}$ and $\tau_{p}=$ $1 \times 10^{-6} \mathrm{~s}$ [15], and direct recombination set by $C_{D I R}=$ $7.2 \times 10^{-10} \mathrm{~cm}^{-3} \cdot \mathrm{s}^{-1}$ [15]. As depicted in Fig. 4(a), the IB energy level inside the bandgap adds two additional possible transitions at $\Delta E_{C I}=0.48 \mathrm{eV}$ and $\Delta E_{I V}=0.95 \mathrm{eV}$, which both contribute to the optical absorption.

The IB is modeled using an effective DOS of $N_{I B}=5 \times$ $10^{16} \mathrm{~cm}^{-3}$ [24], with Gaussian distribution having $\sigma=0.05$ $\mathrm{eV}$ and located at $0.15 \mathrm{eV}$ below the conduction band edge. The charge carrier generation profile is calculated assuming illumination from the anode (p-emitter) and using an occupation dependent model given by

$$
G_{12}=C_{12} \frac{n_{1}}{N_{1}}\left(1-\frac{n_{2}}{N_{2}}\right) .
$$

Here, $C_{12}$ is a constant transition rate from state 1 to state $2, n_{1}$ the carrier density in state 1 and $n_{2}$ the carrier density in state $2, N_{1}$ and $N_{2}$ are the available densities of final states. The IB is coupled to the conduction and valence bands by means of band-to-band rates, similar as in section III-A. We used a capture time of 1 ns from conduction band to intermediate band, and a bimolecular rate constant of $C_{I V}=1 \times 10^{-11} \mathrm{~cm}^{3} \cdot \mathrm{s}^{-1}$ between the valence band and IB.

In Fig. 4(b) we report results of two different sets of simulations that we performed accounting (full lines) and neglecting (dashed lines) the band-occupation for charge carrier generation. In both cases we used constant transition rates of $C_{C I}=C_{I V}=C_{C V}=5 \times 10^{20} \mathrm{~cm}^{-3} \cdot \mathrm{s}^{-1}$, that correspond to an effective absorption coefficient of $1 \times 10^{4} \mathrm{~cm}^{-1}$. It can be observed, that inclusion of the occupation dependency in the IB absorption terms is important, as it considerably reduces the effective absorption. In fact, $J_{S C}$ decreases from 44 (full lines) to $29.3 \mathrm{~mA} \cdot \mathrm{cm}^{-2}$ (dashed lines), which is still higher than the value of the GaAs reference cell [26]. The decrease of $J_{S C}$ and $V_{O C}$ are due respectively to the minor carrier density available for transport because of the electrostatic profile variation and to the introduction of a narrow- 


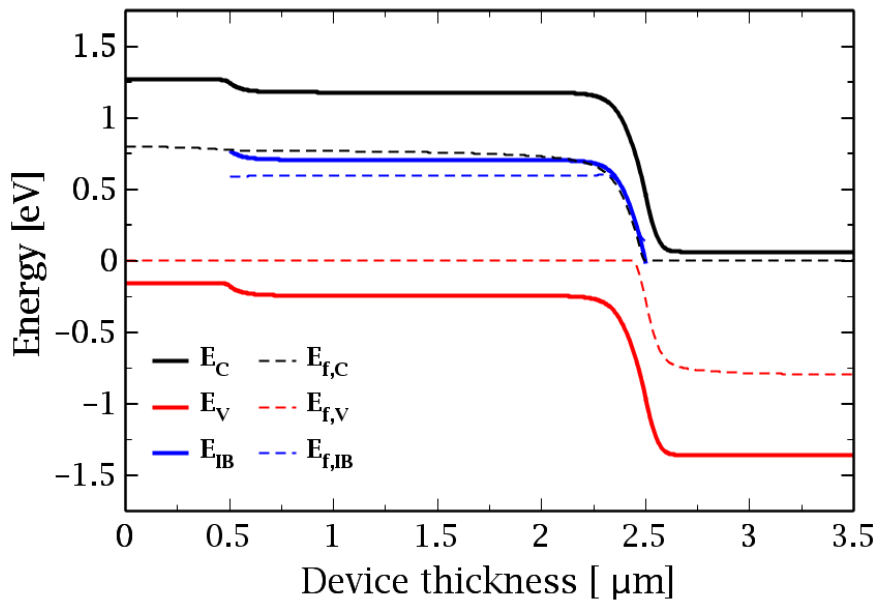

(a)

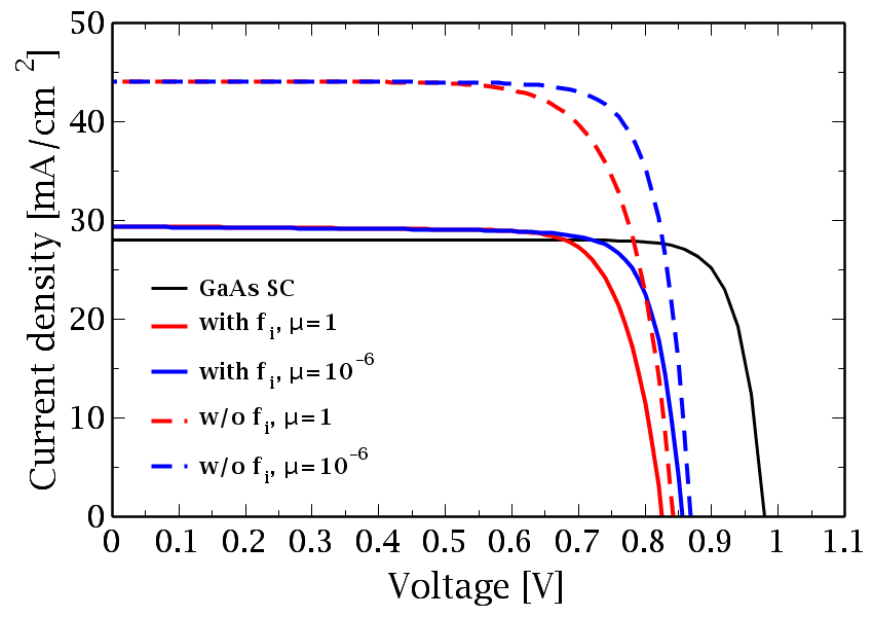

(b)

Fig. 4. (a) Band diagrams in short-circuit current condition for the p-i-n structure simulated. $\boldsymbol{E}_{\boldsymbol{C}}, \boldsymbol{E}_{\boldsymbol{V}}$ and $\boldsymbol{E}_{\boldsymbol{I} B}$ are energy levels and $\boldsymbol{E}_{f, C}, \boldsymbol{E}_{f, \boldsymbol{V}}$ and $\boldsymbol{E}_{\boldsymbol{f}, \boldsymbol{I} \boldsymbol{B}}$ are their respective quasi Fermi levels; (b) JV characteristics comparison between experimental results on GaAs solar cell [27] and on IBSC simulations with different values of $\mu_{I B}$ and generation modeled as: full lines using constant generation, while dashed lines using the occupation dependent generation model.

gap effective material. Both these effects are in accordance with experimental results achieved by Blokhin et al. [27]. Furthermore, we investigate the impact of different mobilities of electrons within the IB (from $10^{-6}$ to $1 \mathrm{~cm}^{2} \cdot \mathrm{V}^{-1} \cdot \mathrm{s}^{-1}$ ). The results shown in Fig. 4(b) indicate that the IB mobility affects the $V_{o c}$ of the cell. Interestingly, a larger mobility decreases $V_{o c}$. This can be interpreted such that at high IB mobility photogenerated carriers will be transported preferably in the IB, which has higher carrier density. Therefore, part of the electrons are extracted towards the contact through the IB, instead of being promoted to the $\mathrm{CB}$, leading to an overall loss of $V_{o c}$.

Note that such a result cannot be obtained when treating the IB by means of trap states in a standard DD model, as done e.g. in [24], which can not consider transport in the IB. Thus, we conclude that the classical DD approach can only simulate the limit case of vanishing transport in the IB, while the multi-particle DD allows going beyond this limitation, even

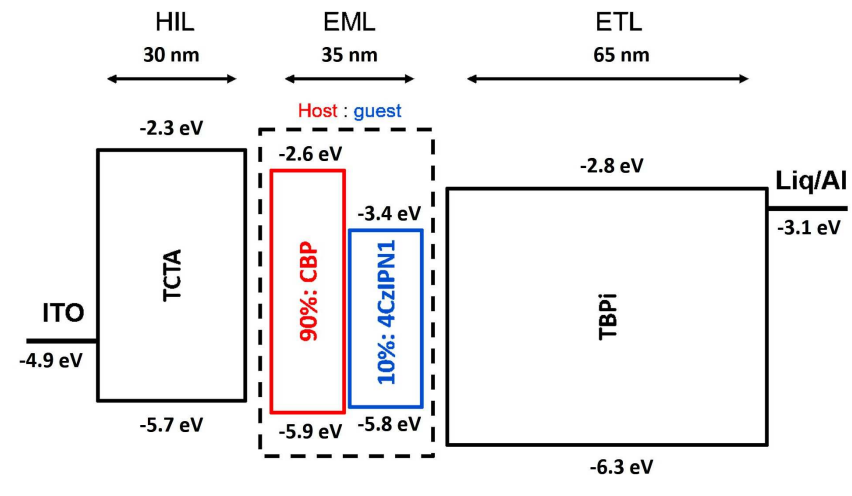

(a)

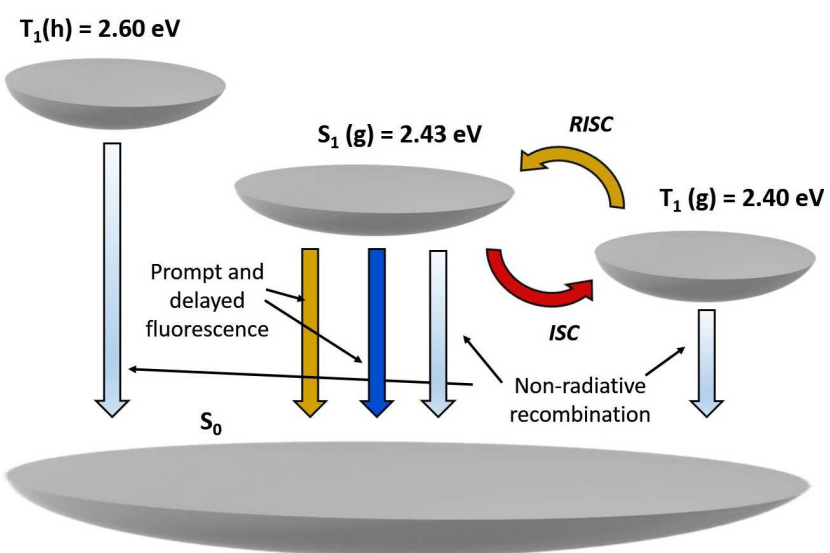

(b)

Fig. 5. (a) Energy levels and work-functions of the simulated TADF OLED stack; (b) Representation of exciton states and recombination rate constants accounted for within the host-guest system of the emitter.

accounting for more additional sub-bands.

\section{Thermally Activated Delayed Fluorescence OLED}

Thermally activated delayed fluorescence (TADF) materials have shown great potential for efficient OLEDs [28]. The triplet recycling via reverse inter-system crossing (RISC) mechanism allows to have metal-free organic fluorescent molecule able to overcome its intrinsic limit of $25 \%$, by harvesting both singlets and triplets exciton states, indicated respectively by $S_{1}(\mathrm{~g})$ and $T_{1}(\mathrm{~g})$. This mechanism occurs in the TADF emitter when the lifetime of triplets is sufficiently long and, at the same time, if the singlet-triplet energy gap is small enough (in the order of few $k_{B} T$ ) to promote the triplet into singlet conversion by thermal activation. Therefore, the total fluorescence is given by two contributions: the prompt fluorescence due to radiative decay of the singlets and the delayed fluorescence given by the emission of singlets generated through RISC. With the aim to solve both carriers and excitons transport for a TADF OLED we simulated the $130 \mathrm{~nm}$ thick stack reported in Fig. 5, where the emitter layer (EML) is made by an host-guest system.

Electron and hole mobilities are provided depending on the region and on the material, using either field-assisted [29] or constant mobility models as reported in Table I. 
TABLE I

CHARGED CARRIER MOBILITY PARAMETERS.

\begin{tabular}{|l|l|l|l|l|l|}
\hline Layer & Material & Holes & Electrons & Units & Ref. \\
\hline HIL & TCTA & $\mu=2 \times 10^{-5}$ & $\mu=1 \times 10^{-7}$ & $\mathrm{~cm}^{2} / \mathrm{Vs}$ & {$[30]$} \\
\hline EML & CBP & $\mu_{0}=3.8 \times 10^{-4}$ & $\mu_{0}=4 \times 10^{-4}$ & $\mathrm{~cm}^{2} / \mathrm{Vs}$ & {$[31]$} \\
(host) & & $E_{0}=1.63 \times 10^{5}$ & $E_{0}=1.82 \times 10^{7}$ & V/cm & \\
\hline EML & 4 CzIPN & $\mu_{0}=7 \times 10^{-5}$ & $\mu_{0}=1.1 \times 10^{-5}$ & $\mathrm{~cm}^{2} / \mathrm{Vs}$ & {$[32]$} \\
(guest) & & $E_{0}=2.7 \times 10^{5}$ & $E_{0}=3.81 \times 10^{5}$ & $V / \mathrm{cm}$ & \\
\hline ETL & TBPi & $\mu=1 \times 10^{-7}$ & $\mu=5 \times 10^{-5}$ & $\mathrm{~cm}^{2} / \mathrm{Vs}$ & {$[33]$} \\
\hline
\end{tabular}

Concerning the neutral carriers, the model accounts for excitons of both host and guest materials. Singlet and triplet states of guest have $E_{S}(g)=2.43 \mathrm{eV}$ and $E_{T}(g)=2.40$ $\mathrm{eV}$ that make $\Delta E_{S T}=0.03 \mathrm{eV}$ suitable to guarantee the activation of TADF process. Differently, for host material we account only for the triplets with $E_{T}(h)=2.60 \mathrm{eV}$, neglecting the singlets because of their rather high energy such as $E_{S}(h)=3.00 \mathrm{eV}$. Considering all the charge and neutral carries, the simulation is performed accounting 8 different charged (see Table I) and 3 neutral (see Fig. 5a) particles with their own Fermi levels. All main kinetic mechanisms included in the simulations involving excitons are schematically reported in Fig. 5(b). Non-radiative recombination in the host is set by $\tau_{n r, T}(h)=1 \times 10^{-5} \mathrm{~s}$, while for guest material both non-radiative and radiative recombinations are included setting characteristic lifetimes $\tau_{n r, S}(g)=1 \times 10^{-5}$ $\mathrm{s}, \tau_{n r, T}(g)=1 \times 10^{-5} \mathrm{~s}$ and $\tau_{r, S}(g)=1 \times 10^{-8} \mathrm{~s}$. The intersystem crossing (ISC) is modeled by a coupling of singlet and triplet populations according to

$$
R_{I S C}=C_{I S C} n_{S}\left(1+\frac{n_{T}}{N_{T}}\right)\left[1-\exp \left(\frac{E_{f, T}-E_{f, S}}{k_{B} T}\right)\right]
$$

where we have set the rate constant to $C_{I S C}=3 \times 10^{6}$ $\mathrm{s}^{-1}$. Note that this model includes RISC as the reverse process of ISC, and that the former will dominate whenever $E_{f, T}>$ $E_{f, S}$. As described in section II-B, the empirical factor $1+$ $n_{T} / N_{T}$ accounts for the dependency on the occupation of the final states. The sign here is positive, though, since we assume excitons to follow Bose-Einstein rather than FermiDirac statistics.

All charged and neutral carrier energy states are distributed according to Gaussian DOS with $N_{0}=1 \times 10^{20} \mathrm{~cm}^{-3}$, with characteristic disorders of $\sigma_{\mathrm{n}, \mathrm{p}}=0.1 \mathrm{eV}$ and $\sigma_{\text {exciton }}=$ $0.05 \mathrm{eV}$, respectively. Exciton generation is modeled by a bimolecular electron-hole recombination term, using a rate constant of $C_{G}=1 \times 10^{-13} \mathrm{~cm}^{-3} \cdot \mathrm{s}^{-1}$ and respecting the $1: 3$ ratio between singlets and triplets.

In accordance with experimental results reported by Baldo et al. [16] we included in simulations the most important bimolecular degradation processes such as triplet-to-triplet annihilation (TTA) and triplet-to-polaron quenching (TPQ), modeled as

$$
\begin{aligned}
& R_{T T A}=C_{T T A} n_{T}^{2}\left[1-\exp \left(-\frac{2 E_{f, T}}{k_{B} T}\right)\right] \\
& R_{T P Q}=C_{T P Q} n_{T} n_{Q}\left[1-\exp \left(-\frac{E_{f, T}}{k_{B} T}\right)\right] .
\end{aligned}
$$

In the former, two triplets interact leading to the loss of one of them, while in the latter a triplet vanishes interacting with a polaron. The quencher, which can be an electron or a hole, is indicated by the index $Q$. In our case we assumed TPQ only due to holes.

The rate constants are set under the assumption to have percentage losses for TTA and TPQ of $15 \%$ and $30 \%$, respectively, at $6 \mathrm{~V}$ operation. The resulting parameters for host and guest materials are $C_{T T A, h}=5 \times 10^{-10} \mathrm{~cm}^{-3} \cdot \mathrm{s}^{-1}, C_{T T A, g}=$ $5 \times 10^{-9} \mathrm{~cm}^{-3} \cdot \mathrm{s}^{-1}, C_{T P Q, h}=2 \times 10^{-12} \mathrm{~cm}^{-3} \cdot \mathrm{s}^{-1}$ and, $C_{T P Q, g}=6 \times 10^{-12} \mathrm{~cm}^{-3} \cdot \mathrm{s}^{-1}$.

Results in Fig. 6 provide a clear understanding of the importance of RISC on the radiative emission within the emitter. Figure 6(a) shows a comparison between the overall radiative recombination (black line) and the RISC rate (red line) within the EML. The inclusion of singlet-triplet coupling in guest material improves the total emission by $\sim 20 \%$. Figure 6(b) reports the internal quantum efficiency (IQE) at different operation voltage. As expected, while the nonradiative contribution is insensitive to the polarization voltage, both TPQ and TTA mechanisms, especially the latter, lead to IQE drop for increasing drive current.

\section{CONCLUSIONS}

We have presented an implementation of a generalized multi-particle drift-diffusion model capable to overcome the limitations imposed by the standard drift-diffusion model based on two-carrier transport equations. The modeling approach provides the possibility to take into account multiple carrier sub-populations, each assumed in a thermal local equilibrium, and guarantees a strictly thermodynamically consistent formulation for recombination-generation between populations. We have demonstrated its ability and flexibility to describe systems with different application contexts. First we have reproduced the thermionic emission for a metalsemiconductor interface modeling a typical Schottky contact, successfully matching results obtained using the classical DD and finding the expression of the effective transfer rate $C_{n n^{\prime}}$ as functional parameter of Richardson constant $\left(A^{*}\right)$.

Then we have investigated the ability of the model on the calculation of charge transport within the intermediate band for a GaAs-based IBSC device. Exploiting a band-occupation dependent generation model we have investigated the impact of the additional sub-band absorption contributions on the device operation. Results obtained on the $J_{s c}$ increase and $V_{O C}$ decrease than GaAs solar cell baseline are in accordance with experimental results found in literature.

Finally, we have simulated a TADF OLED device explicitly treating charge carrier and exciton sub-populations with the aim to analyze singularly all mechanisms which determine the optical emission. The model allowed us to calculate the 


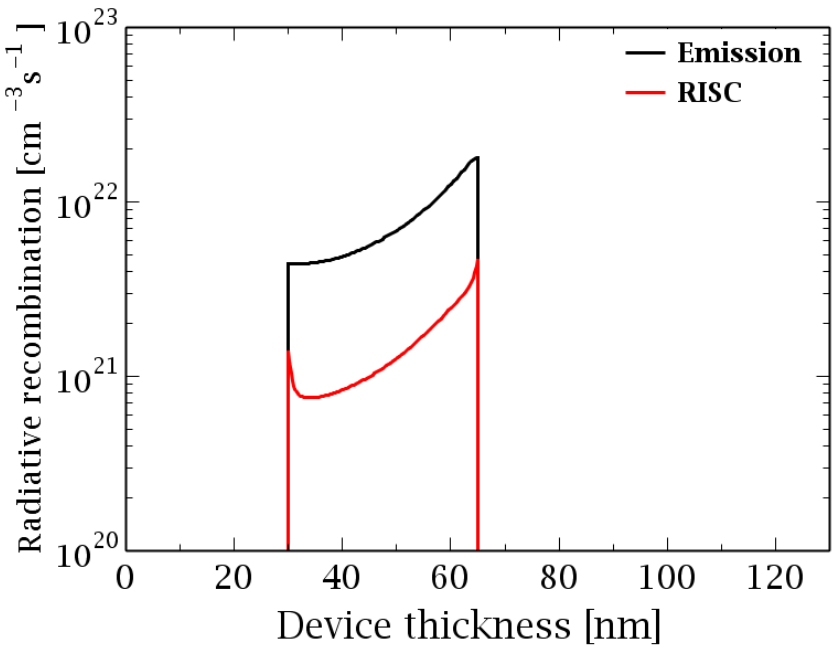

(a)

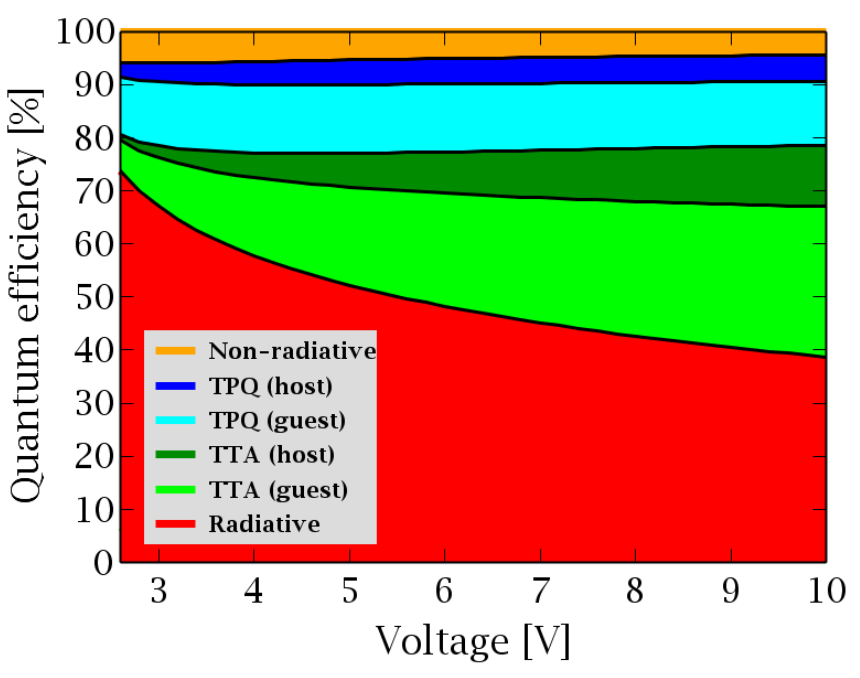

(b)

Fig. 6. (a) Radiative emission (black) and RISC (red) within the emitter at $6 \mathrm{~V}$ operation; (b) Quantum efficiency contributions given by nonradiative recombination (orange), triplet-to-polaron quenching (blue and cyan), triplet-to-triplet annihilation (light and dark green) and the IQE given by radiative emission (red) at different voltage operation calculated with $\mathrm{mp}-\mathrm{DD}$.

emission profile and to estimate every single radiative and nonradiative recombination contribution during the device operation, especially the thermally activated delayed fluorescence contribution given by the triplet recycling.

The model is not limited to the electronic particle but could describe on equal footing ions opening to a consistent solution of system were electrons and ions play a fundamental role in the device behavior (electrochemical device, dye sensitized solar cells, fuel cells, chemical storage devices, etc.).

\section{ACKNOWLEDGMENT}

We acknowledge the funding from the European Unions Horizon 2020 research and innovation programme under grant agreement No. 737089 (CHIPSCOPE) and No. 826013 (IMPRESSIVE).

\section{REFERENCES}

[1] W. v. Roosbroeck, "Theory of the flow of electrons and holes in germanium and other semiconductors," Bell Syst. Tech. J., vol. 29, no. 4, pp. 560-607, 1950, doi: 10.1002/j.1538-7305.1950.tb03653.x.

[2] H. Kosina and M. Nedjalkov, "The Monte Carlo method for semiclassical charge transport in semiconductor devices," Math. Comput. Simul., vol. 55, no. 1, pp. 93 - 102, 2001, the Second IMACS Seminar on Monte Carlo Methods, doi: 10.1016/S0378-4754(00)00249-4.

[3] W. Kaiser, J. Popp, M. Rinderle, T. Albes, and A. Gagliardi, "Generalized kinetic Monte Carlo framework for organic electronics," Algorithms, vol. 11, no. 4, p. 37, 2018, doi: 10.3390/a11040037.

[4] R. Coehoorn, H. van Eersel, P. Bobbert, and R. Janssen, "Kinetic Monte Carlo study of the sensitivity of OLED efficiency and lifetime to materials parameters," Adv. Funct. Mater., vol. 25, no. 13, pp. 2024 2037, 2015, doi: 10.1002/adfm.201402532.

[5] M. Yoshida, E. Arai, H. Nakamura, and Y. Terunuma, "Excess vacancy generation mechanism at phosphorus diffusion into silicon," J. Appl. Phys., vol. 45, no. 4, pp. 1498-1506, 1974, doi: 10.1063/1.1663450.

[6] F. Römer and B. Witzigmann, "Effect of Auger recombination and leakage on the droop in InGaN/GaN quantum well LEDs," Opt. Express, vol. 22, no. S6, pp. A1440-A1452, 2014, doi: 10.1364/OE.22.0A1440.

[7] M. Kantner, M. Mittnenzweig and T. Koprucki, "Hybrid quantumclassical modeling of quantum dot devices," Phys. Rev. B, vol. 96, no. 20, pp. 205301, 2017, doi: 10.1103/PhysRevB.96.205301.

[8] S. Selberherr, Analysis and simulation of semiconductor devices. Springer Science \& Business Media, 2012, doi: 10.1007/978-3-70918752-4.

[9] L. D. Landau, E. M. Lifshitz and L. P. Pitaevskii, Electrodynamics of continuous media, vol. 8. Elsevier, 2013, isbn: 9780750626347.

[10] J. S. Blakemore, Semiconductor Statistics. Dover Publications, New York, 1987, isbn: 9781483148946.

[11] L. D. Landau and E. M. Lifshitz, Statistical Physics. Number v. 5. Elsevier Science, 2013, isbn: 9780080570464.

[12] M. Lundstrom, Fundamentals of carrier transport. Cambridge university press, 2009, isbn: 9780521631341.

[13] J. C. Kim and J. P. Wolfe, "Bose-Einstein statistics of an excitonic gas in two dimensions: Excitons and biexcitons in a GaAs quantum well,' Phys. Rev. B, vol. 57, no. 16, pp. 9861, 1998, doi: 10.1007/s10825-0110377-4.

[14] P. A. Markowich, C. A. Ringhofer, and C. Schmeiser, "From kinetic to fluid dynamical models," in Semiconductor Equations. Springer, 1990, pp. 83-103.

[15] Y. Varshni, "Band-to-band radiative recombination in groups IV, VI, and III-V semiconductors (i)," Phys. Status Solidi B, vol. 19, no. 2, pp. 459-514, 1967, doi: 10.1002/pssb.19670190202.

[16] M. A. Baldo, C. Adachi, and S. R. Forrest, "Transient analysis of organic electrophosphorescence. II. Transient analysis of triplet-triplet annihilation," Phys. Rev. B, vol. 62, pp. 10967-10977, Oct 2000, doi: 10.1103/PhysRevB.62.10967.

[17] R. Coehoorn, L. Zhang, P. Bobbert, and H. Van Eersel, "Effect of polaron diffusion on exciton-polaron quenching in disordered organic semiconductors," Phys. Rev. B, vol. 95, no. 13, p. 134202, 2017, doi: 10.1103/PhysRevB.95.134202.

[18] S. M. Sze and K. K. Ng, Physics of semiconductor devices. John wiley \& sons, 2006

[19] M. A. der Maur, G. Penazzi, G. Romano, F. Sacconi, A. Pecchia, and A. D. Carlo, "The multiscale paradigm in electronic device simulation," IEEE Trans. Electron. Dev., vol. 58, no. 5, pp. 1425-1432, May 2011, doi: 10.1109/TED.2011.2114666.

[20] C. Crowell, "The Richardson constant for thermionic emission in Schottky barrier diodes," Solid-State Electronics, vol. 8, no. 4, pp. 395 - 399, 1965, doi: 10.1016/0038-1101(65)90116-4.

[21] W. Shockley and H. J. Queisser, "Detailed balance limit of efficiency of p-n junction solar cells," J. Appl. Phys., vol. 32, no. 3, pp. 510-519, 1961, doi: 10.1063/1.1736034.

[22] A. Luque and A. Martí, "Increasing the efficiency of ideal solar cells by photon induced transitions at intermediate levels," Phys. Rev. Lett., vol. 78, pp. 5014-5017, Jun 1997, doi: 10.1103/PhysRevLett.78.5014.

[23] _ _ "The intermediate band solar cell: Progress toward the realization of an attractive concept," Adv. Mat., vol. 22, no. 2, pp. 160-174, 2010, doi: 10.1002/adma.200902388.

[24] K. Yoshida, Y. Okada, and N. Sano, "Self-consistent simulation of intermediate band solar cells: Effect of occupation rates on device characteristics," Appl. Phys. Lett., vol. 97, no. 13, p. 133503, 2010, doi: $10.1063 / 1.3488815$ 
[25] I. Vurgaftman, J. Meyer, and L. Ram-Mohan, "Band parameters for IIIV compound semiconductors and their alloys," J. Appl. Phys., vol. 89, no. 11, pp. 5815-5875, 2001, doi: 10.1063/1.1368156.

[26] Y. Okada, T. Morioka, K. Yoshida, R. Oshima, Y. Shoji, T. Inoue, and T. Kita, "Increase in photocurrent by optical transitions via intermediate quantum states in direct-doped InAs/GaNAs strain-compensated quantum dot solar cell," J. Appl. Phys., vol. 109, no. 2, p. 024301, 2011, doi: 10.1063/1.3533423.

[27] S. Blokhin, A. Sakharov, A. Nadtochy, A. Pauysov, M. Maximov, N. Ledentsov, A. Kovsh, S. Mikhrin, V. Lantratov, S. Mintairov Semiconductors, "AlGaAs/GaAs photovoltaic cells with an array of InGaAs QDs," Semiconductors, vol. 43, no. 4, pp. 514-518, 2009, doi: $10.1134 /$ S1063782609040204.

[28] H. Uoyama, K. Goushi, K. Shizu, H. Nomura, and C. Adachi, "Highly efficient organic light-emitting diodes from delayed fluorescence," $\mathrm{Na}$ ture, vol. 492, no. 7428, p. 234, 2012, doi: 10.1038/nature11687.

[29] H. Bässler, "Charge transport in disordered organic photoconductors a Monte Carlo simulation study," Phys. Status Solidi B, vol. 175, no. 1, pp. 15-56, 1993, doi: 10.1002/pssb.2221750102.

[30] D.-H. Lee, Y.-P. Liu, K.-H. Lee, H. Chae, and S. M. Cho, "Effect of hole transporting materials in phosphorescent white polymer lightemitting diodes," Org. Electron., vol. 11, no. 3, pp. 427 - 433, 2010, doi: 10.1016/j.orgel.2009.11.022.

[31] N. Matsusue, Y. Suzuki, and H. Naito, "Charge carrier transport in neat thin films of phosphorescent iridium complexes," Jpn. J. Appl. Phys., vol. 44, no. 6R, p. 3691, 2005, doi: 10.1143/JJAP.44.3691.

[32] S. Wang, Y. Zhang, W. Chen, J. Wei, Y. Liu, and Y. Wang, "Achieving high power efficiency and low roll-off OLEDs based on energy transfer from thermally activated delayed excitons to fluorescent dopants," Chem. Commun., vol. 51, no. 60, pp. 11972-11975, 2015, doi: $10.1039 / \mathrm{c} 5 \mathrm{cc} 04469 \mathrm{c}$

[33] J.-H. Jou, S. Kumar, A. Agrawal, T.-H. Li, and S. Sahoo, "Approaches for fabricating high efficiency organic light emitting diodes," J. Mater. Chem. C, vol. 3, no. 13, pp. 2974-3002, 2015, doi: $10.1039 / \mathrm{C} 4 \mathrm{TC} 02495 \mathrm{H}$ 